\title{
Understanding the regulation of surfactant gene expression
}

\author{
W. Jacot, J. Bousquet
}

Among a number of biochemical and morphological criteria, alveolar type-II epithelial cells are defined by the synthesis of surfactant proteins. These molecules are implicated in numerous human diseases such as neonatal respiratory distress syndrome [1] and possibly acute respiratory distress syndrome (ARDS) [2], where a better understanding could lead to new therapeutic modalities via a targeted induction of surfactant synthesis. The synthesis of surfactant proteins (SPs) is under the control of a variety of potential developmental and hormonal regulators, however, two transcription factors, thyroid transcription factor (TTF)-1 and hepatocyte nuclear factor (HNF)-3, have a crucial role in mediating the expression of SPs [3]. The promoter region of the SP-B gene has been studied extensively [4]. In the human SP-B gene, a region located at the immediate 5 ' flanking region of the basal promoter TATA box between $80-110$ contains two cis-acting elements for TTF-1 and HNF-3 binding [4]. These two elements are important for the specificity and activation of SPs gene expression in the lung.

SP-B is a 79-amino acid peptide critical to postnatal respiratory adaptation. SP-B is the only surfactant-associated protein required for postnatal lung function and survival. Complete deficiency of SP-B in mice and humans results in lethal neonatal respiratory distress syndrome and is characterised by a virtual absence of lung compliance, highly disorganised lamellar bodies, and greatly diminished levels of SP-C mature peptide [5]. Its expression is developmentally regulated. Although the expression of SP-B is known to be dependent, at least in part, on TTF-1 and HNF-3, other factors such as SP1 and SP3 are also required for its expression. TTF-1 can also regulate SP-A and SP-C expression in mammals. Besides TTF-1, human upstream stimulatory factor-1, nuclear factor (NF)-1, and GT-box binding protein also play roles in regulating SP-A and/or SP-C expression [3].

TTF-1 also known as Nkx2.1, is a homeodomain-containing transcription factor which plays a role in regulating genes expressed within the thyroid, lung and brain, including thyroglobulin, thyroid peroxidase, Clara cell secretory protein (CCSP), type-I pneumocyte-specific T1 gene and the SPs [6]. Additional studies, including gene-targeting experiments, have clearly shown that expression of TTF-1 is essential for morphogenesis of the thyroid, lung and ventral forebrain, as TTF-1 knockout mice lack these organs.

There are three primary domain structures in TTF-1: an $\mathrm{N}$-terminal transactivation domain, a deoxyribonucleic acid (DNA)-binding homeodomain in the middle, and a C-terminal activation domain [7]. The N-terminal domain was shown to

Clinique des Maladies Respiratoires and U-454 Institut National de la Santé et de la recherche médicale (INSERM), Hôpital Arnaud de Villeneuve, University Hospital, Montpellier, France.

Correspondence: J. Bousquet, Clinique des Maladies Respiratoires, Hôpital Arnaud de Villeneuve, Centre Hospitalier Universitaire, 34295 Montpellier Cedex 5, France. Fax: 33 467042708. E-mail: bousquet@montp.inserm.fr bear the main transactivation activity because the mutant without the N-terminal domain completely abolished the transcriptional activity whereas the mutant with the C-terminus deleted only partially reduced activity. Structurally, it has been suggested that the N-terminal domain has functional properties similar to the typical transactivation acidic domain in VP16. The characteristics of this domain imply that this may be the region where TTF-1 interacts with other potential factors/cofactors to regulate responsive gene expression in vivo.

Furthermore, during the development of mouse lung, both TTF-1 and HNF-3 are expressed early at the onset of lung growth (gestational day 10) and throughout foetal lung development, but SP-B expression is not detected until a later gestational stage (gestational day 17) [8]. This suggests that TTF-1 and/or HNF-3 alone cannot fully account for the differential regulation of SP-B gene expression during the development. An additional cofactor(s) or the modified form of TTF-1, therefore, might be required in regulating the SP-B gene expression in vivo. It is thus important to know the different modulators of this critical transcriptional regulator. Transcription factors are often regulated by associated protein cofactors that are able to modify their activity by several different mechanisms. Some of theses modulators have been previously described, such as calreticulin [9], increasing the steady-state concentration of active TTF-1 protein in the cell, retinoic acid receptor alpha [10] via the enhancement of TTF-1 homeodomain DNA binding activity to a hSP-B enhancer oligonucleotide, and TTF-2 [11] which is able to repress TTF-1 activity in a DNA-binding independent way. These proteins are able to modulate TTF-1 transactivation activity by formation of protein complexes. Other methods for the modulation of TTF-1 activity are to prevent its nuclear translocation via post-translational modifications such as phosphorylation, for example under transforming growth factor (TGF)- $\beta$ exposition [12],or redox status [13], oxidation decreasing TTF-1 DNA binding activity.

In a previously published study, using a modified yeast, YANG et al. [3] screened a human lung complementary DNA (cDNA) library for DNA-binding proteins, other than TTF-1 and HNF-3, that interacted with the promoter region of human SP-B gene previously identified as critical for the tissue-specific expression of this gene. A novel $26 \mathrm{kDa}$ polypeptide, BR22, was identified that activated the reporter gene expression in yeast. This gene is expressed in many tissues and encodes a protein with bipartite nuclear localisation signals. Further in vitro and in vivo analyses indicated that BR22 could form a protein complex with TTF-1 and synergistically activate the SP-B promoter in mammalian cells.

In this issue of the European Respiratory Journal, YANG et al. [14] demonstrate that BR22, now designated as TAP26, can be detected as a $34 \mathrm{kDa}$ polypeptide in H441 and 293 cells lysates. Co-immunoprecipitation of the endogenous TTF-1/ TAP26 complex was achieved in vivo, suggesting an in vivo stable association of these two compounds. An immunohistochemical staining of TAP26 on lung sections showed a 
TAP26 expression in SP-B producing alveolar epithelial cells. In addition, TAP26 was found to be coordinately expressed with SP-B and TTF-1 in adult lung, foetal lung, type-II pneumocytes and H441 cells, and to be induced by two known SP-B inducers, namely dexamethasone and cyclic adenosine monophosphate (cAMP). All these data suggest that TAP26 is an associated protein of thyroid transcription factor-1 in the lung alveolar epithelial cells where surfactant protein gene expressions take place in vivo, suggesting a co-activator role of TAP26 in the regulation of the surfactant gene expression.

Knowledge of the different factors regulating surfactant proteins gene expression and synthesis could be of great scientific and clinical relevance in surfactant-associated diseases. Surfactant therapy has revolutionised neonatal care and is used routinely for preterm infants with respiratory distress syndrome. Surfactant-replacement therapy may have a role in preterm neonates with bronchopulmonary dysplasia, meconium aspiration syndrome and pneumonia [15]. Commercial surfactant preparations currently available are not optimal because of the variability of surfactant protein content and their susceptibility to inhibition. Further progress in the treatment of neonatal respiratory disorders may include the development of "designer" surfactant preparations or the induction of the surfactant synthesis by alveolar type-II epithelial cells. Since TAP26 expression is modulated coordinately with surfactant protein $\mathrm{B}$ and thyroid transcription factor-1, a new research area of possible therapeutic interest has been opened by the paper of YANG et al. [14]. However, there are still a number of issues to be addressed including the mechanisms by which TAP26 and thyroid transcription factor-1 interacts.

\section{References}

1. Jobe AH. Glucocorticoids, inflammation and the perinatal lung. Semin Neonatol 2001; 6: 331-342.

2. Leikauf GD, McDowell SA, Wesselkamper SC, et al. Acute lung injury: functional genomics and genetic susceptibility. Chest 2002; 121: Suppl. 3, 70S-75S.

3. Yang YS, Yang MC, Wang B, Weissler JC. BR22, a novel protein, interacts with thyroid transcription factor-1 and activates the human surfactant protein B promoter. Am $\mathrm{J}$ Respir Cell Mol Biol 2001; 24: 30-37.

4. Venkatesh VC, Planer BC, Schwartz M, Vanderbilt JN, White RT, Ballard PL. Characterization of the promoter of human pulmonary surfactant protein B gene. Am J Physiol 1995; 268: L674-L682.

5. Weaver TE, Conkright JJ. Function of surfactant proteins B and C. Annu Rev Physiol 2001; 63: 555-578.

6. Bingle CD. Thyroid transcription factor-1. Int $J$ Biochem Cell Biol 1997; 29: 1471-1473.

7. Tell G, Perrone L, Fabbro D, et al. Structural and functional properties of the $\mathrm{N}$ transcriptional activation domain of thyroid transcription factor-1: similarities with the acidic activation domains. Biochem $J$ 1998; 329: 395-403.

8. Zhou L, Lim L, Costa RH, Whitsett JA. Thyroid transcription factor-1, hepatocyte nuclear factor-3beta, surfactant protein $\mathrm{B}, \mathrm{C}$, and Clara cell secretory protein in developing mouse lung. J Histochem Cytochem 1996; 44: 1183-1193.

9. Perrone L, Tell G, Di Lauro R. Calreticulin enhances the transcriptional activity of thyroid transcription factor- 1 by binding to its homeodomain. J Biol Chem 1999; 274: 46404645.

10. Yan C, Naltner A, Conkright J, Ghaffari M. Protein-protein interaction of retinoic acid receptor alpha and thyroid transcription factor-1 in respiratory epithelial cells. $J$ Biol Chem 2001; 276: 21686-21691.

11. Perrone L, Pasca di Magliano M, Zannini M, Di Lauro R. The thyroid transcription factor 2 (TTF-2) is a promoterspecific DNA-binding independent transcriptional repressor. Biochem Biophys Res Commun 2000; 275: 203-208.

12. Kumar AS, Gonzales LW, Ballard PL. Transforming growth factor-beta (1) regulation of surfactant protein B gene expression is mediated by protein kinase-dependent intracellular translocation of thyroid transcription factor-1 and hepatocyte nuclear factor 3. Biochim Biophys Acta 2000; 1492: 45-55.

13. Arnone MI, Zannini M, Di Lauro R. The DNA binding activity and the dimerization ability of the thyroid transcription factor I are redox regulated. J Biol Chem 1995; 270: 12048-12055.

14. Yang M-CW, Wang B, Weissler JC, Margraf LR, Yang Y-S. $\mathrm{BR} 22$, a $26 \mathrm{kDa}$ thyroid transcription factor-1 associated protein (TAP26), is expressed in human lung cells. Eur Respir J 2003; 22: 28-34.

15. Merrill JD, Ballard RA. Pulmonary surfactant for neonatal respiratory disorders. Curr Opin Pediatr 2003; 15: 149-154. 